\author{
Marquette University \\ e-Publications@Marquette
}

Electrical and Computer Engineering Faculty Research and Publications

Electrical and Computer Engineering,

Department of

$10-9-2013$

\title{
Microelectromechanical Systems (MEMS) Resistive Heaters as Circuit Protection Devices
}

Ronald A. Coutu Jr.

Marquette University, ronald.coutu@marquette.edu

Scott A. Ostrow

United State Air Force

Follow this and additional works at: https://epublications.marquette.edu/electric_fac

Part of the Computer Engineering Commons, and the Electrical and Computer Engineering Commons

\section{Recommended Citation}

Coutu, Ronald A. Jr. and Ostrow, Scott A., "Microelectromechanical Systems (MEMS) Resistive Heaters as Circuit Protection Devices" (2013). Electrical and Computer Engineering Faculty Research and

Publications. 326.

https://epublications.marquette.edu/electric_fac/326 
Marquette University

\section{e-Publications@Marquette}

\section{Electrical and Computer Engineering Faculty Research and Publications/College of Engineering}

This paper is NOT THE PUBLISHED VERSION; but the author's final, peer-reviewed manuscript. The published version may be accessed by following the link in the citation below.

IEEE Transactions on Components, Packaging and Manufacturing Technology, Vol. 3, No. 12, (October, 2013). DOI. This article is (C) Institute of Electrical and Electronic Engineers (IEEE) and permission has been granted for this version to appear in e-Publications@Marquette. IEEE does not grant permission for this article to be further copied/distributed or hosted elsewhere without the express permission from IEEE.

\section{Contents}

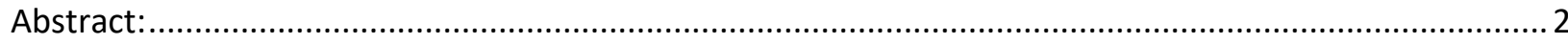

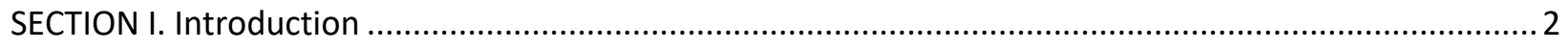

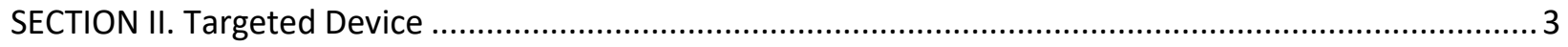

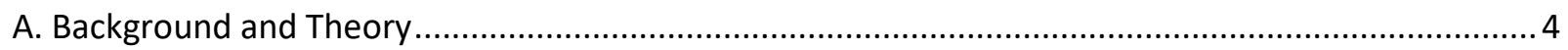

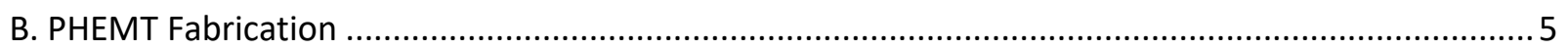

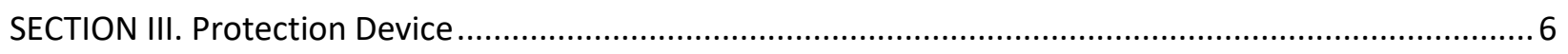

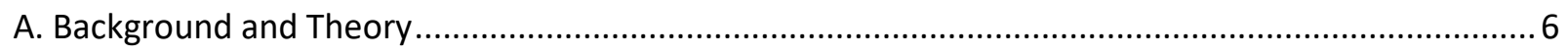

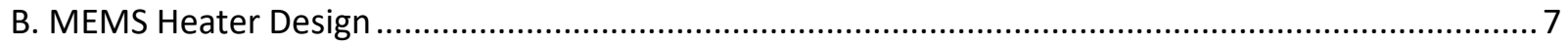

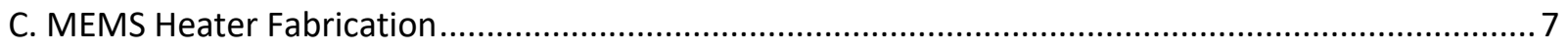

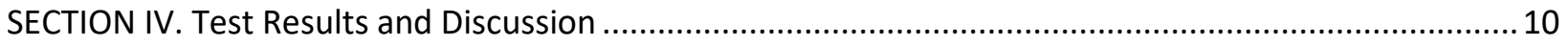

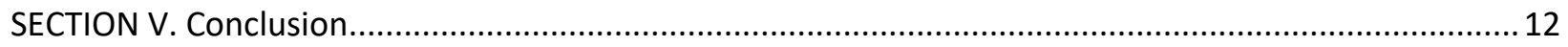

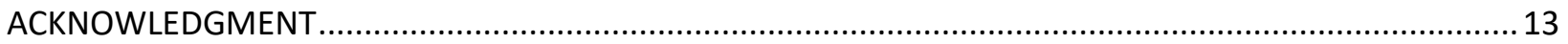




\title{
Microelectromechanical Systems (MEMS) Resistive Heaters as Circuit Protection Devices
}

\author{
Ronald A. Coutu
}

Department of Electrical Engineering, the Air Force Institute of Technology, Wright-Patterson Air Force Base, $\mathrm{OH}$

\author{
Scott. A Ostrow
}

United States Air Force, Air Force Institute of Technology, Wright-Patterson Air Force Base, $\mathrm{OH}$

\section{Abstract:}

With increased opportunities for the exploitation (i.e., reverse engineering) of vulnerable electronic components and systems, circuit protection has become a critical issue. Circuit protection techniques are generally software-based and include cryptography (encryption/decryption), obfuscation of codes, and software guards. Examples of hardware-based circuit protection include protective coatings on integrated circuits, trusted foundries, and macro-sized components that self-destruct, thus destroying critical components. This paper is the first to investigate the use of microelectromechanical systems (MEMS) to provide hardware-based protection of critical electronic components to prevent reverse engineering or other exploitation attempts. Specifically, surface-micromachined polycrystalline silicon to be used as meandering resistive heaters were designed analytically and fabricated using a commercially available MEMS prototyping service (i.e., PolyMUMPs), and integrated with representative components potentially at risk for exploitation, in this case pseudomorphic high-electron mobility transistors (pHEMTs). The MEMS heaters were initiated to self-destruct, destroying a critical circuit component and thwart a reverse engineering attempt. Tests revealed reliable self-destruction of the MEMS heaters with approximately $25 \mathrm{~V}$ applied, resulting in either complete operational failure or severely altering the pHEMT device physics. The prevalent failure mechanism was metallurgical, in that the material on the surface of the device was changed, and the specific failure mode was the creation of a short-circuit. Another failure mode was degraded device operation due to permanently altered device physics related to either dopant diffusion or ohmic contact degradation. The results, in terms of the failure of a targeted electronic component, demonstrate the utility of using MEMS devices to protect.

\section{SECTION I. Introduction}

With increased opportunities for the exploitation of electronic systems, the use of circuit protection methods has become vitally important. ${ }^{1,2}$ Attacks on systems and individual devices can include physical/side-channel attacks and logical attacks. Physical attacks, which deal with exploiting the implementation of a system, include invasive attacks such as microprobing and reverse engineering (RE), and noninvasive attacks such as power analysis, electromagnetic analysis, timing attacks, and fault 
induction. ${ }^{3}$ Logical attacks, which deal with observing the response of a system to stimuli, include software attacks, such as viruses and exploiting crypto/protocol weaknesses in a system. ${ }^{3,4}$ Circuit protection techniques are generally software-based and include cryptography (encryption/decryption), obfuscation of codes, layouts and data flows, and software guards. ${ }^{5-6,7,8}$ Examples of hardware-based circuit protection include protective coatings on integrated circuits, entire product enclosures, trusted processors and foundries, and the introduction of complexity into circuit design and macro-sized selfdestructing components that protect critical circuits by destroying them prior to exploitation..$^{5-6,7,9,10}$ Current state of the art in hardware-based circuit protection is passive camouflage of individual components so that they visually appear to be another standard component (i.e., a nand gate looks like a nor gate). ${ }^{11}$ This approach prevents RE when adversaries create logic flow diagrams directly from physical circuits without actually applying power and measuring signals.

This paper presents the first-ever microelectromechanical systems (MEMS) resistive heater used as a circuit protection device. Resistive heaters utilize the concepts of joule heating and thermal transfer, when a voltage is applied, to heat up and/or burn out electronic components that they are placed or fabricated on top of. The approach for this demonstration was to utilize a commercial MEMS fabrication process to create prototype polycrystalline silicon meandering and spiral resistive heater devices and test their effectiveness for providing circuit protection against RE via the self-destruction of critical circuit components. We use unpackaged pseudomorphic high-electron-mobility transistors (pHEMTs) to represent key circuit components potentially at risk for exploitation. pHEMTs were chosen because they are high-performance devices, commonly used in military radio frequency (RF) systems, and are a special form of modulation-doped field-effect transistors (MODFETs) or high-electron-mobility transistors (HEMTs), where a thin lattice matching layer is used to isolate defects from the substrate. ${ }^{12}$ When considering the self-destruction or degradation of potentially exploitable components, the electronic failure modes and failure mechanisms need to be considered.

Failure modes are the symptoms which highlight that a failure has occurred, typically seen in the degradation or change in a device's response parameters-voltage, current, resistance, etc. Standard failure modes are open circuits, short-circuits, functional failures, and degraded performance. These failure modes can be influenced by a number of variables including, but not limited to, component design and geometry, material doping levels, humidity, and temperature. Temperature is commonly seen as the variable that has the biggest influence on inducing and increasing the likelihood of failure.

Failure mechanisms are the means by which a device or component degrades or stops working. These mechanisms can be chemical, environmental, metallurgical, physical, or any combination of them. ${ }^{13,14}$ In this paper, failure modes were triggered thermally by placing MEMS resistive heaters directly onto devices potentially targeted for exploitation -in this case pHEMTs.

\section{SECTION II. Targeted Device}

The pHEMT device shown in Fig. 1, which was fabricated as part of an advanced semiconductor devices course, was used to represent a vulnerable electronic component at risk for exploitation. 


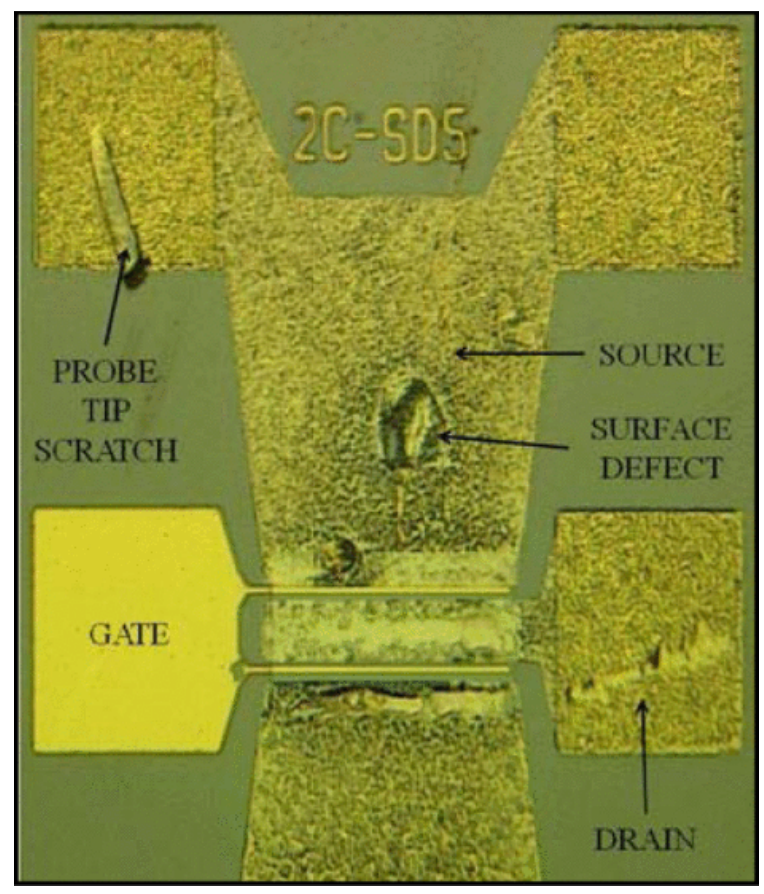

Fig. 1. Optical image showing a representative pHEMT device used in this paper. The surface damage was a result of rapid thermal annealing during fabrication and did not affect the proper operation of the device.

\section{A. Background and Theory}

Field-effect transistors (FETs) are widely used in switching and amplification applications. They are characterized by having three terminals, where one terminal controls the flow of carriers (electrons or holes) in the channel that links the other two terminals, thus controlling the current between those terminals. In FETs, these terminals are referred to as the drain, source, and gate, with the gate being the terminal that controls the flow of carriers from the source terminal to the drain terminal. This current is controlled by a voltage at the gate and the formation of a gate capacitor, which creates the channel between the other two terminals. ${ }^{14,15}$ FETs are classified at a high level by the formation of the gate capacitor, with junction FETs (JFETs) utilizing the depletion layer in a p-n junction, metal-semiconductor FETs (MESFETs) utilizing the depletion layer in a Schottky junction, and insulated-gate FETs (IGFETs) using an insulator material. The pHEMT falls under the IGFET classification, which can be subdivided further into metal-oxide-semiconductor FETs (MOSFETs), metal-insulator-semiconductor FETs (MISFETs), and heterojunction FETs (HFETs). These classifications are based on how the insulator is formed, with MOSFETs having an oxide layer grown as the insulator, MISFETs having a dielectric deposited as the insulator, and HFETs having an insulator based on the deposition of a high-bandgap material to form a heterojunction with the channel layer. The pHEMT falls under the HFET classification, which is composed of MODFETs (i.e., HEMTs) and heterojunction insulated-gate FETs (HIGFETs). The distinction between these two classifications is based on whether the high-bandgap material is doped (MODFETs) or undoped (HIGFETs).

A standard way to assess the performance of an FET is through its current-voltage $(I-V)$ characteristics, which are derived from the basic operation of the device. By plotting the drain current $I_{D}$ versus the drain voltage $V_{D}$, and varying the gate voltage $V_{G}$, a set of curves can be obtained to highlight this 
operation, as shown in Fig. 2 for a pHEMT used in this paper. The data plotted was collected using an Alessi REL-6100 probe station and the HP4155A semiconductor parameter analyzer.

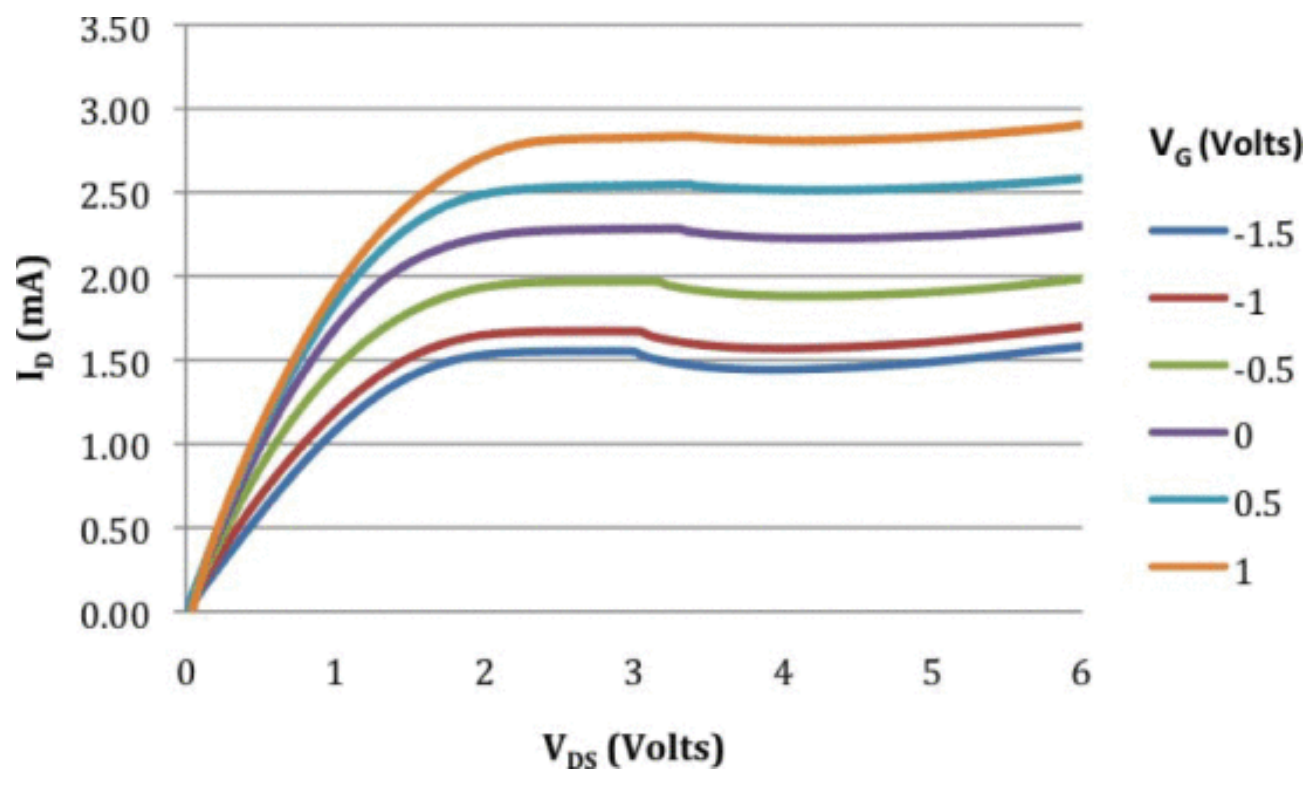

Fig. 2. Graph showing a representative pHEMT baseline $I-V$ characteristic collected using an Alessi REL6100 probe station and an HP4155A semiconductor parameter analyzer. These curves highlight that the baseline prior to the placement of the self-destruct device is operating as designed.

At a specific applied gate voltage, the drain current will be proportional to the drain voltage over a range of low $V_{D}$ values, referred to as the linear region. Eventually, there is a point where this linear relationship breaks down, referred to as the nonlinear region, and the applied $V_{D}$ starts to cause the channel at the drain end to shrink in size. What is known as pinch-off occurs when the channel is essentially closed off at the drain end of the channel. Past this point is referred to as the saturation region, and the drain current essentially remains constant even if the drain voltage is increased. ${ }^{15}$

Focusing further on the MODFET configuration, the high-bandgap material is doped, such as n-doped AlGaAs, while the channel layer underneath is undoped, forming the heterojunction. A quantum well, referred to as a two-dimensional electron gas (2-DEG), forms from the creation of this heterojunction, in which electrons from the doped-layer conduction band diffuse toward the undoped layer. This occurs because the doped layer has a wider energy bandgap than the undoped layer, and the shifting electrons are confined to the 2-DEG region as a result of band bending. The formation of the 2-DEG results in a channel that is free of impurity scattering, and as a result has high-mobility carriers traversing through the channel. This is known as modulation doping and it is where MODFETs get their name from. ${ }^{12,14,15}$

\section{B. PHEMT Fabrication}

The pHEMT devices were fabricated on GaAs substrates with a thin epitaxial lattice-match layer of $\mathrm{In}_{\mathrm{x}} \mathrm{Ga}_{1-\mathrm{x}}$ As between the GaAs substrate and the AlGaAs layer to increase electron mobility. A straightforward three-mask process based on standard fabrication steps were followed, resulting in pHEMT devices with T-shaped gates. ${ }^{16}$ 


\section{SECTION III. Protection Device}

In this paper, MEMS meandering and spiral resistive heaters are examined as circuit protection devices for pHEMTs. The design concept was to disrupt device operation and/or alter device physics by generating a sufficiently high temperature gradient directly on top of an unpackaged pHEMT. The background, design, and fabrication of the MEMS devices are discussed next.

\section{A. Background and Theory}

Thermal, or heat, transfer occurs when a temperature gradient exists in a material. The mechanisms for heat transfer are thermal conduction, natural thermal convection, forced thermal convection, and radiation. Typically, when considering MEMS devices, conduction is emphasized, while radiation and convection are de-emphasized since most often the devices are hermetically sealed. If the MEMS devices are not sealed, convection would require more consideration. ${ }^{17,18}$

Thermal conduction involves the movement of heat in a solid as a result of a temperature gradient. It is characterized by the agitation of molecules in a material, with energy transferring from fast moving particles to slower moving particles, while the material itself remains motionless. ${ }^{17,18}$

The heat transfer takes place in the direction of the negative of the temperature gradient, moving from a high to a low temperature. The thermal conductivity and temperature gradient, therefore, will determine the rate of heat transfer for a particular material. ${ }^{18}$

Thermal transfer is dictated by the thermal resistance of the material. The higher the thermal resistance, the lower the heat transfer rate, which results in greater thermal isolation. Thermal resistance is the ratio of the temperature difference to the heat flow, and is analogous to electrical resistance in the following form:

$R=\rho \times(l / A)$

where $\rho$ is the electrical resistivity, $I$ is the length, and $A$ is the surface area of a resistor. The power loss associated with a current flowing through a resistor is

$P=I^{2} \times R$

where $I$ is the current and $R$ is the resistance. This power loss is also referred to as joule or ohmic heating. ${ }^{17}$

Resistors are simple multifunctional devices that can be used for biasing electronic components as well as functioning as test structures for evaluating material properties. They are adaptable because they can be fabricated from a wide range of materials, such as polysilicon, diffused bulk silicon, silicon-oninsulator (SOI), and dielectrics, as well as with a range of applications, such as thermal sensors, resistive heaters, and piezoresistors. The resistance of a fabricated resistor is determined by its length, width, thickness, resistivity, sheet resistance, and the quality of material it is made of. ${ }^{13}$

Meandering resistors are compact and typically used when linear resistors would negatively impact circuit designs due to limited design space or fabrication area. A concern for square meandering 
resistors, however, is that the current density at each corner block or bend is not evenly distributed and therefore contributes less to the overall resistance, and so a correction factor is required..$^{20}$ Equation (3) accounts for this effect and is used to predict the resistance of square meandering resistive heaters

(3)

$R=R_{\mathrm{s}} \times\left(N+\left(k^{\prime} \times N_{\mathrm{cb}}\right)\right)$

where $R$ is the overall resistance, $R_{\mathrm{s}}$ is the sheet resistance, $N$ is the number of subcomponent blocks in the straight sections, $k$ 'is the corner block correction factor, and $N \mathrm{cb}$ is the number of corner blocks. ${ }^{20}$

\section{B. MEMS Heater Design}

The following were the primary considerations for this demonstration: 1) use a well understood surrogate device for the demonstration; 2) use a resistive heater to generate a sufficiently large thermal gradient for disrupting device operation or altering device physics; and 3) allow for postmortem analysis and investigation. pHEMTs were used to address consideration 1, while MEMS devices were used to address considerations 2 and 3.

Specifically, MEMS heaters were designed to maximize the pHEMT surface area, reach the maximum temperature with a $25 \mathrm{~V}$ circuit protect signal applied, and minimize potential resistor hot spot failures due to current crowding. Two heater variations were designed to address the surface area design constraint and the current crowding concern. The square meandering resistors, designed using (3), provided a compact design with the highest surface area coverage and consisted of 17 arms (500 $\mu \mathrm{m}$ long, $20 \mu \mathrm{m}$ wide) with 10- $\mu \mathrm{m}$ gaps between the arms. Current crowding (common in the corner blocks of meandering resistors) and premature device failure concerns inspired the circular or spiral resistor variation that was designed using $\underline{(1)}$. The circular geometry ( $\sim 350 \mu$ m overall radius) was investigated to minimize potential current crowding effects by using a relatively large radius of curvature structure. In both design variations, a 1.5- $\mu \mathrm{m}$-thick, polysilicon layer with known resistivity $(\sim 33.3 \Omega \cdot \mathrm{cm})$ and sheet resistance $(\sim 22 \Omega / \square)$ was used. The corresponding resistance of each resulting structure (square and spiral) was approximately equal (i.e., $\sim 650 \Omega$ ). Finally, the use of MEMS heaters for this demonstration facilitated heater removal and allowed reliable postmortem analysis of the pHEMT devices.

\section{MEMS Heater Fabrication}

The Polysilicon MultiUser MEMS Process (PolyMUMPs) is a commercially available polycrystalline silicon (i.e., polysilicon) surface micromachining process that offers two releasable polysilicon layers and one layer fixed to the substrate. The process consists of 12 photolithography mask steps and consists of seven total layers all fabricated on top of a $<100>$ oriented $n$-type silicon wafer. ${ }^{21}$ The first layer, namely silicon nitride, is used for electrical isolation between the substrate and the subsequent polysilicon mechanical layers. The oxide films (two in total) are the sacrificial layers, while the polysilicon films (three in total) are the structural and fixed layers. The final evaporated gold layer is for electrical contact pads, electrical wiring, and creating reflective surfaces. ${ }^{21}$

Devices with and without probe pads were designed and fabricated using PolyMUMPs. The devices with probe pads were tested on chip by applying a voltage across the device to characterize how the MEMS 
device initiated and eventually burned out or self-destructed. The devices without probe pads were designed with smaller anchors; so, once released, they could be manually detached from the die using a micromanipulator probe station. Once detached, the center of the heater was placed over the transistor gate region, using a probe, and optically aligned using fabrication alignment marks and other device features. The meandering heaters were easily aligned, whereas the spiral heaters proved more challenging to align on top of the pHEMTs. Although this manual technique of heater placement was effective for this proof-of-concept demonstration and postmortem analyses, an automated flip-bonding approach would be needed for manufacturing actual integrated circuits protected using this method.

Figs. 3 and 4 show scanning electron microscopy (SEM) images, prior to on-chip testing, of a square meandering and a spiral resistive heater, respectively.

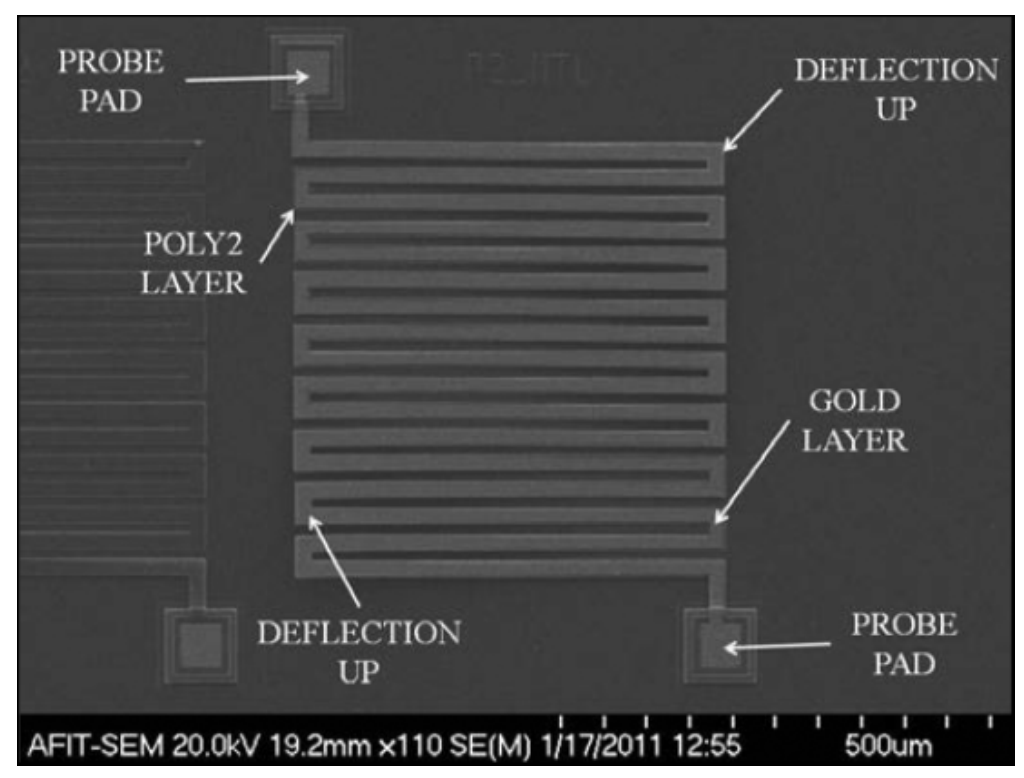

Fig. 3. Scanning electron microscopy (SEM) image of a meandering resistive heater with probe pads for testing and characterization prior to integration with a pHEMT device. 


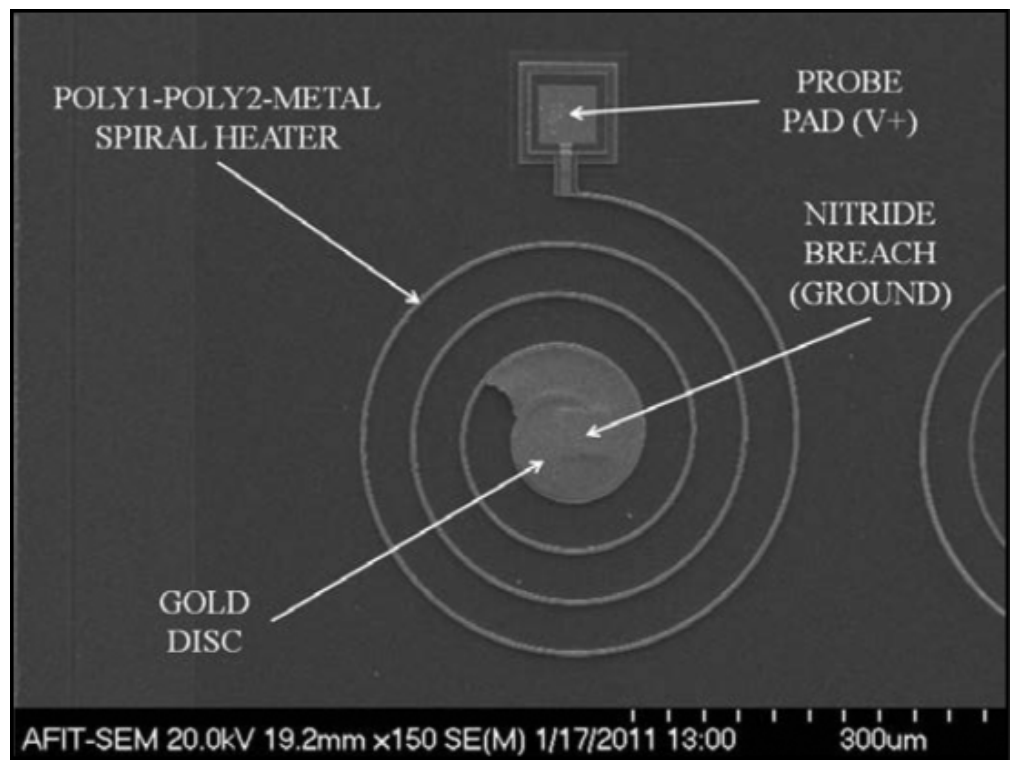

Fig. 4. Scanning electron microscopy (SEM) image of a polycrystalline silicon spiral heater fabricated with a metal layer that was not completely lifted off, thus highlighting the prototyping nature of the PolyMUMPs process.

Fig. 4 also shows a portion of the metal layer in the center of the spiral heater that was not completely lifted off during fabrication, thus highlighting the prototyping nature of the PolyMUMPs process.

Fig. 5 shows an example of a square meandering resistive heater that was removed from a PolyMUMPs die and placed on top of a surrogate pHEMT device.

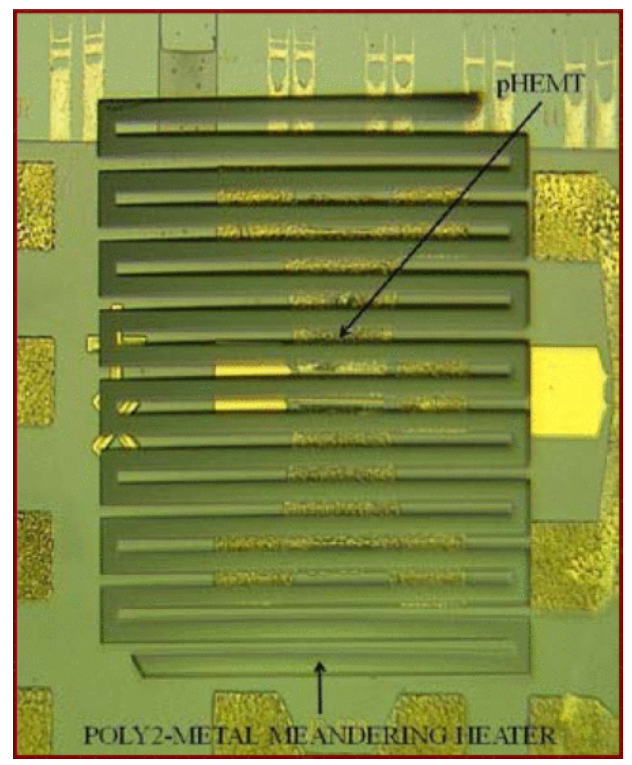

Fig. 5. Optical picture of a square meandering resistive heater placed on a surrogate pHEMT. The meandering prototype heater was removed from a PolyMUMPs chip and placed on top of the pHEMT device. 


\section{SECTION IV. Test Results and Discussion}

Prior to the circuit protection demonstration, individual meandering (see Fig. 3) and spiral (see Fig. 4) heater devices, with probe pads, were extensively tested by applying dc voltages and characterized using an OptoTherm micro-thermal imaging system. As expected, the effect of applying a dc selfdestruct signal similarly caused catastrophic damage to both meandering and circular resistive heaters. Additionally, no thermal image hot spots, due to current crowding in the meandering resistor corner blocks, were observed.

Next, unmodified pHEMT devices were tested and baselined in terms of their measured $I-V$ characteristics as shown in Fig. 2 . Finally, pHEMT devices were fitted with meandering resistive heaters and spiral resistive heaters.

The modified pHEMT devices were then tested, before applying the circuit protect signal, to verify baseline functionality, and then again after removing the resistive heaters, to verify PHEMT damage. The meandering heaters, shown in Fig. 6, self-destructed and evaporated with the application of approximately $25 \mathrm{~V}$.

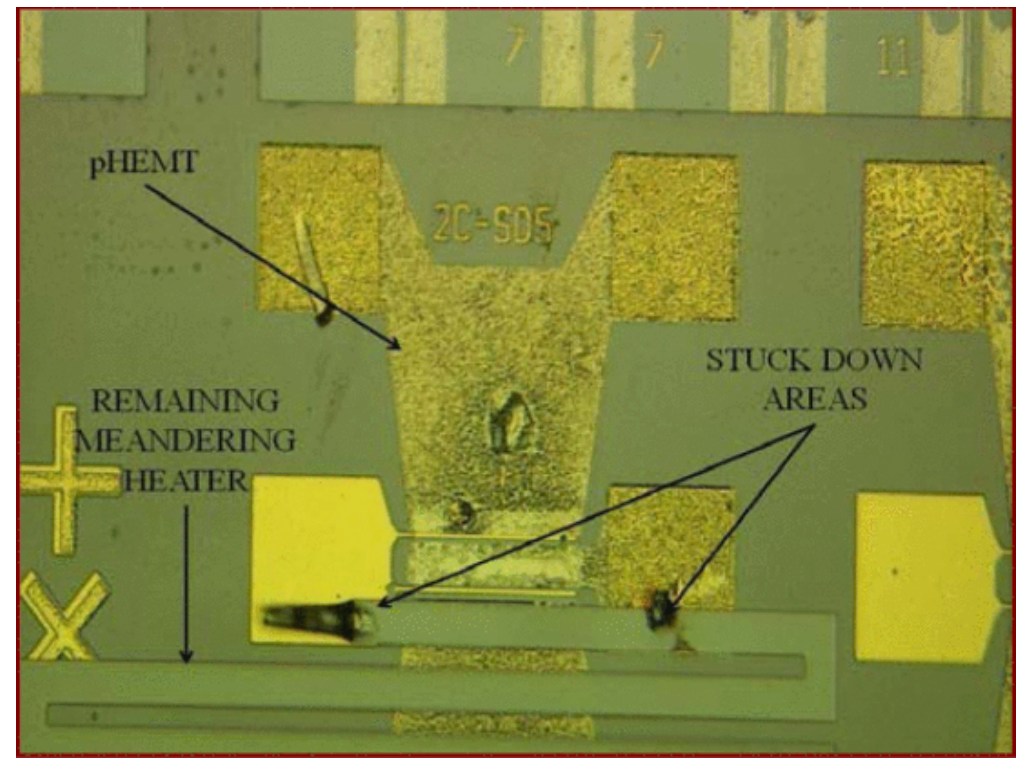

Fig. 6. Optical image and postmortem analysis of a meandering resistive heater that was stuck on top of a pHEMT device after ignition. A voltage was applied to the meandering heater, which caused it to heat up and burn out, resulting in permanently altered device physics of the underlying PHEMT device.

In each case, the pHEMT devices failed and no longer exhibited useable performance, based on $I-V$ characteristic results. The initiation of the heaters either short-circuited the device terminals or permanently altered the physics of the component, thus destroying normal functionality of the targeted circuit. During postmortem analyses (after removing the MEMS heaters), it was discovered that initiated MEMS heaters primarily caused short-circuits across the device terminals. Figs. 7 and 8 are SEM images highlighting a short-circuit across the drain-gate-source of a representative pHEMT device. Additionally, Fig. 8 clearly shows the depletion, migration, and accumulation of material. 


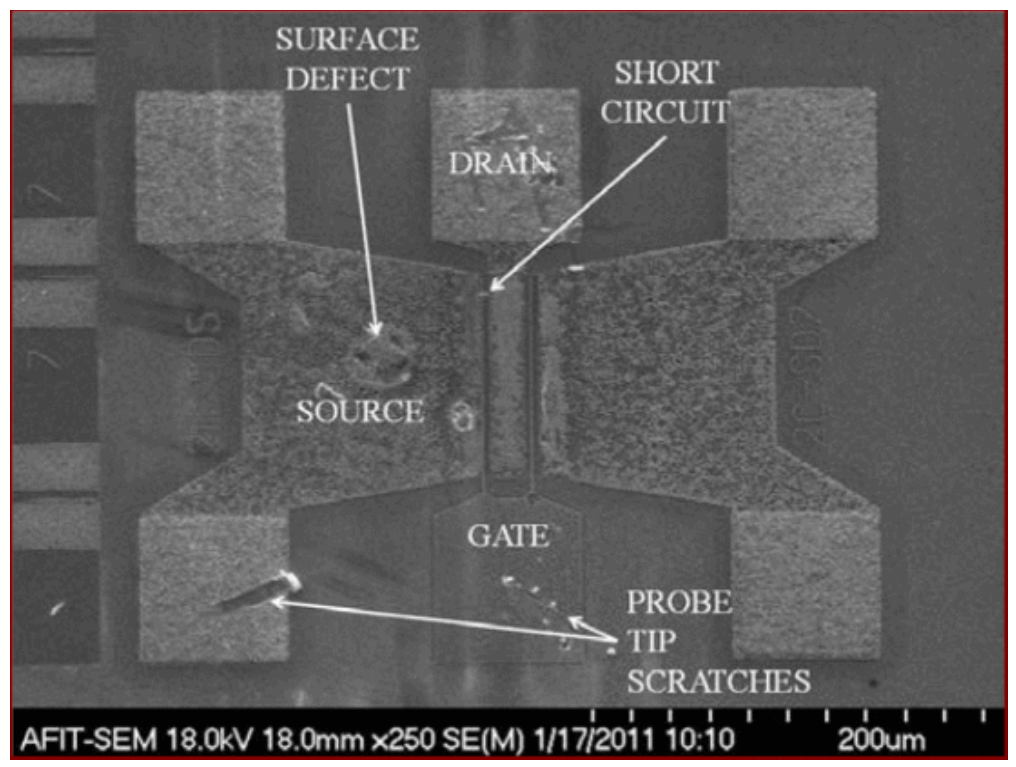

Fig. 7. Scanning electron microscopy (SEM) image of the effects of initiating of a meandering resistive heater on a pHEMT device. A short-circuit, labeled above, was formed across the drain-gate-source of the pHEMT.

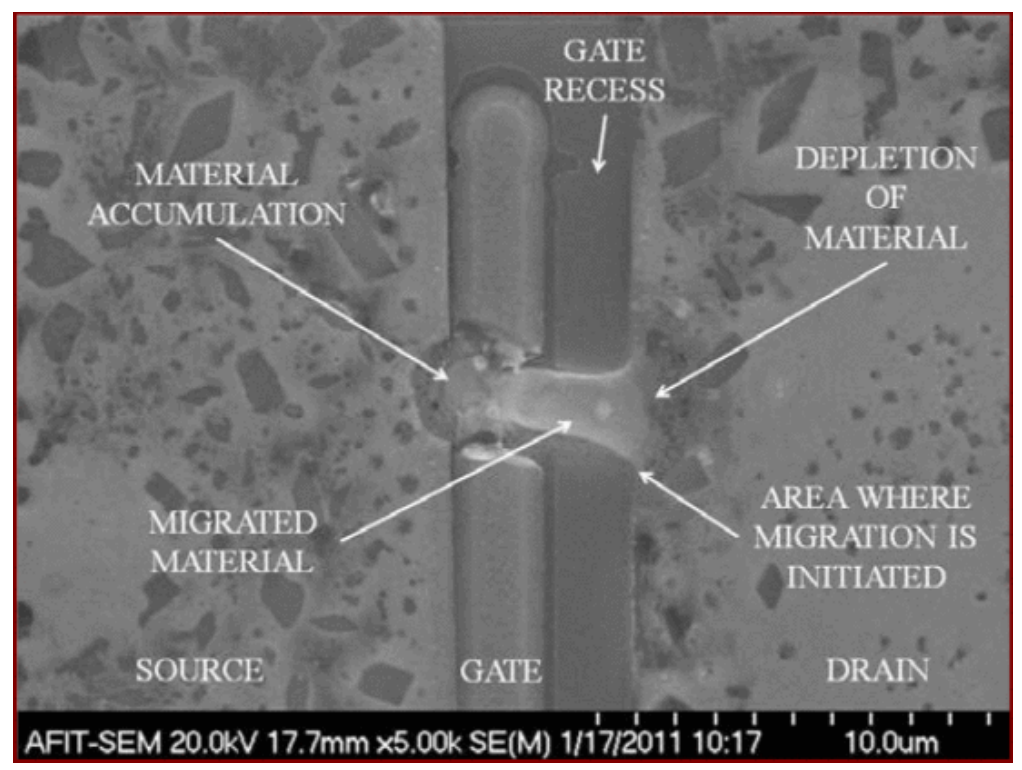

Fig. 8. Scanning electron microscopy (SEM) image of a short-circuited pHEMT as a result of operating a meandering resistive heater self-destruct sequence. The image shows that material has migrated from the drain to the gate and the source, thus causing a short-circuit.

Fig. 9 is an example of severely degraded device physics characterized by a significantly altered $I-V$ characteristic after the initiation of a spiral heater. 


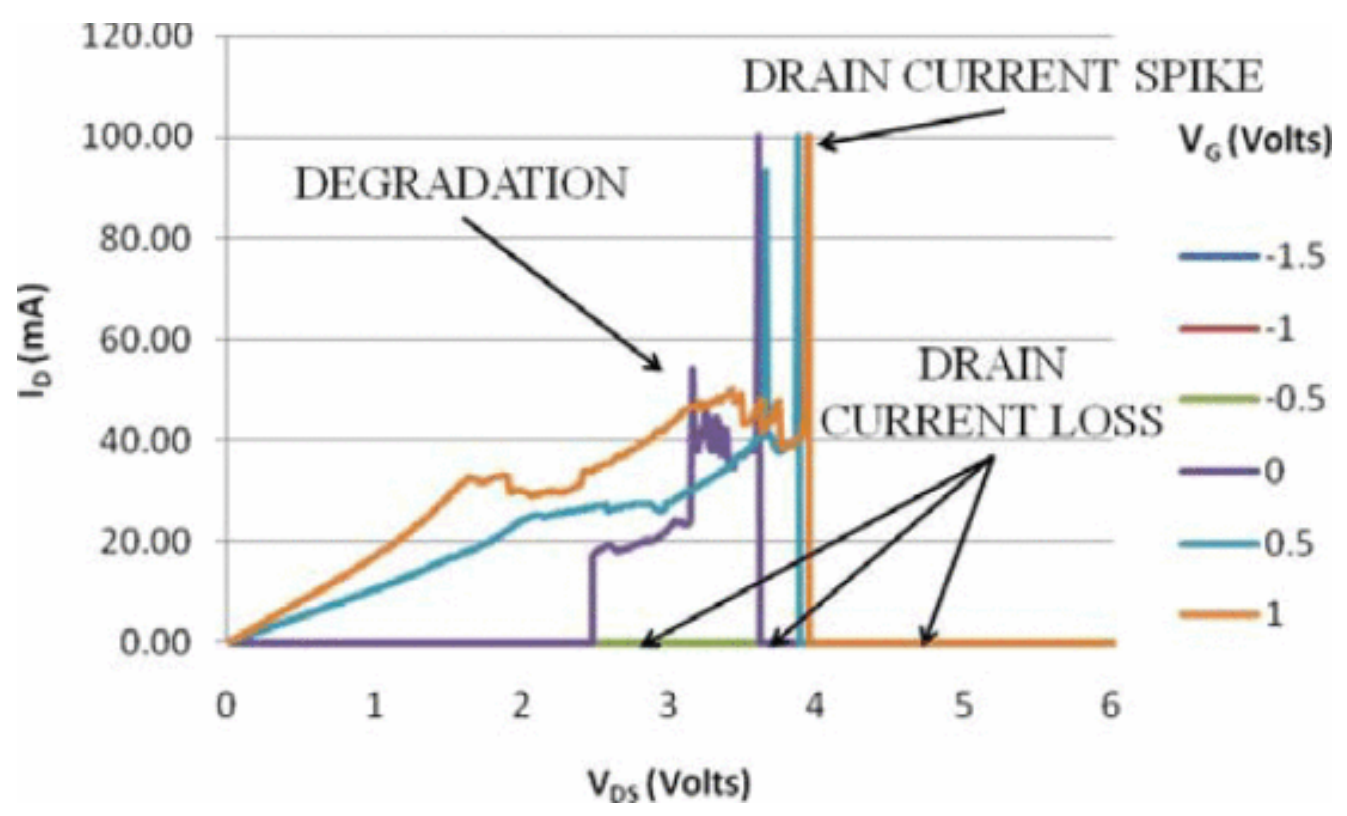

Fig. 9. pHEMT $I-V$ characteristics after initiation and removal of a MEMS spiral resistive heater. There is obvious degradation in device physics, with spikes in the drain current as well as the complete loss of drain current at higher voltages and operating points.

It is apparent from the $I-V$ characteristics, shown in Fig. 9, that the pHEMT was not operating as it did before the spiral heater was initiated. Unlike most of the results, however, the data shown in Fig. 9 did not result in the complete operational failure of a pHEMT device, but rather permanently altered the physics of the device. Potential failure mechanisms for the device illustrated by Fig. 8 include, but are not limited to, unintended diffusion of dopants, degradation of ohmic contacts in the device terminals, and electromigration. ${ }^{14}$ Regardless of the exact pHEMT failure mechanism, the targeted circuitry was again protected by the heater protection circuit.

The results of this demonstration clearly show that by using a MEMS-based self-destruct component, in this case a resistive heater, on top of an electronic component, in this case a pHEMT, the functionality of an otherwise functional component can be completely destroyed, thus protecting targeted circuitry from exploitation.

\section{SECTION V. Conclusion}

This paper has demonstrated how MEMS-based self-destruct components, such as meandering square or spiral resistive heaters, can be effectively used as circuit protection devices for preventing system exploitation. This was evidenced by pHEMT devices failing to operate properly after meandering and circular heaters were placed on top of the device and initiated using a $25 \mathrm{~V}$ circuit protect signal. The advantage of this method is that, by altering device physics or destroying functionality of an individual component, an entire circuit can be protected from RE. In other words, when RE is accomplished, the observed circuit functionality will not be correct because key devices, within the circuit, are not operating properly. This hardware-based approach to circuit protection ensures that intellectual property and mission-critical data are not compromised. Future research will focus on directly fabricating MEMS self-destruct components onto very large scale integrated circuits. 


\section{ACKNOWLEDGMENT}

The authors would like to thank R. Johnston, the AFIT cleanroom technician, for assistance with the pHEMT device fabrication.

\section{References}

1. S. Pope, "Trusted integrated circuit strategy", IEEE Trans. Compon. Packag. Technol., vol. 31, no. 1, pp. 230-233, Mar. 2008.

2. J. Keller, Anti-Tamper Technologies Seek to Keep Critical Military Systems Data in the Right Hands, Apr. 2010.

3. S. Ravi, A. Raghunathan, P. Kocher, S. Hattangady, "Security in embedded systems: Design challenges", ACM Trans. Embedded Comput. Syst., vol. 3, no. 3, pp. 461-491, 2004.

4. J. T. McDonald, Y. C. Kim, D. J. Koranek, J. D. Parham, "Evaluating component hiding techniques in circuit topologies", Proc. IEEE ICC, pp. 1138-1143, 2012-Jun.

5. I. I. Huber, F. Arthur, J. M. Scott, The role and nature of anti-tamper techniques in U.S. defense acquisition, USA, DC, Washington:Dept. Air Force, Acquisition Rev. Quarterly, pp. 268-355, 1999.

6. E. D. Bryant, M. J. Atallah, M. R. Stytz, "A survey of anti-tamper technologies", J. Defense Softw. Eng., vol. 17, no. 11, pp. 12-16, 2004.

7. R. Anderson, M. Kuhn, "Tamper resistance-A cautionary note", Proc. 2nd USENIX Workshop Electron. Commerce, vol. 2, pp. 1-11, 1996.

8. J. English, D. Coe, R. Gaede, D. Hyde, J. Kulick, "MEMS-assisted crytography for CPI protection", IEEE Security Privacy, vol. 5, no. 4, pp. 14-21, Jul.-Aug. 2007.

9. W. L. Gore, Frangible Polymer Circuit Acts as Tamper Sensor, Oct. 2009.

10. J. Grand, "Protecting your crown jewels: An introduction to embedded security for hardware-based products", Comput. Fraud Security, vol. 2005, no. 10, pp. 13-20, Oct. 2005.

11. L. W. Chow, J. P. Baukus, B. J. Wang, R. P. Cocchi, Camouflaging a standard cell based integrated circuit, Apr. 2012.

12. S. M. Sze, Modern Semiconductor Device Physics, USA, NY, New York:Wiley, 1998.

13. S. Franssila, Introduction to Microfabrication, USA, NY, New York:Wiley, 2010.

14. M. Ohring, Reliability and Failure of Electronic Materials and Devices, USA, CA, San Diego:Academic, 1998.

15. S. M. Sze, K. K. Ng, Physics of Semiconductor Devices, USA, NY, New York:Wiley, 2007.

16. R. A. Coutu, Advanced Topics in Microelectronics, USA, OH, Wright Ptrsn:AFIT, 2010.

17. C. Liu, Foundations of MEMS, USA, NJ, Upper Saddle River:Prentice-Hall, 2005.

18. J. P. Holman, Heat Transfer, USA, NY, New York:McGraw-Hill, 2010.

19. M. L. Minges, Electronic Materials Handbook: Packaging, USA, FL, Boca Raton:CRC Press, 1989.

20. M. Pecht, Handbook of Electronic Package Design, USA, FL, Boca Raton:CRC Press, 1991.

21. A. Cowen, B. Hardy, R. Mahadevan, S. Wilcenski, MUMPs Design Handbook, USA, NC, Durham:MEMSCAP, Inc., 2011. 